\title{
Computational Techniques for Motif Search
}

\author{
Sanguthevar Rajasekaran \\ UTC Chair Professor, Dept. of CSE \& Director, Booth Engineering Center for Advanced \\ Technologies, University of Connecticut \\ rajasek@engr.uconn.edu
}

\begin{abstract}
The problem of identifying meaningful patterns (i.e., motifs) from biological data has been studied extensively due to its paramount importance. Motifs are fundamental functional elements in proteins vital for understanding gene function, human disease, and identifying potential therapeutic drug targets. Several versions of the motif search problem have been identified in the literature. Numerous algorithms have been proposed for motif search as well. In this talk we survey some of these algorithms. We also summarize our contributions to motif search and related problems. In addition, we will summarize MnM, a web based system built by us for motif search that is used by biologists widely.
\end{abstract}

